\title{
MOTIVOS DA INTERNAÇÃO POR ORDEM JUDICIAL NA TERAPIA INTENSIVA: PERSPECTIVA DO ENFERMEIRO
}

Cintia Iara Oliveira ${ }^{1}$, Mara Ambrosina de Oliveira Vargas², Edison Devos Barlem³, Sueli Dias de Araújo ${ }^{4}$, Débora Feijó Vieira ${ }^{5}$, Daniela Cardozo ${ }^{6}$

Objetivo: analisar os motivos da internação por ordem judicial na UTI, na perspectiva dos enfermeiros intensivistas. Metodologia: pesquisa qualitativa, exploratória e analítica cujos participantes foram 108 enfermeiros intensivistas, atuantes em UTI nas regiões sudeste e sul do Brasil e que afirmaram já ter vivenciado situações de internação por ordem judicial. Os dados foram coletados por um questionário, no periodo entre abril de 2015 à agosto de 2016 e analisados de acordo com a Análise de Conteúdo. Resultados: foram analisadas as categorias aumento pela demanda pelo serviço de UTI e falta de estrutura institucional e na região da residência do paciente. Conclusões: a internação por ordem judicial, em muitos casos, é o único meio do paciente ter seus direitos garantidos.

Descritores: Enfermagem; Unidade de Terapia Intensiva; Judicialização da saúde; Ética; Hospitalização.

\section{REASONS FOR HOSPITALIZATION BY JUDICIAL ORDER IN INTENSIVE THERAPY: NURSE PERSPECTIVE}

Objective: to analyze the reasons for hospitalization by judicial order in the ICU, from the perspective of intensive care nurses. Methodology: qualitative, exploratory and analytical research whose participants were 108 intensive nurses, working in ICUs in the southeastern and southern regions of Brazil and who have already experienced situations of hospitalization by judicial order. The data were collected by a questionnaire, in the period between April 2015 to August 2016 and analyzed according to the Content Analysis. Results: the categories were analyzed by the increase in demand for the ICU service and lack of institutional structure and in the region of the patient's residence. Conclusions: hospitalization by court order, in many cases, is the only way for patients to have their rights guaranteed.

Descriptors: Nursing; Intensive Care Units; Health's Judicialization; Ethics; Hospitalization.

\section{MOTIVOS DE LA INTERNACIÓN POR ORDEN JUDICIAL EN LA TERAPIA INTENSIVA: PERSPECTIVA DEL ENFERMERO}

Objetivo: analizar los motivos de la internación por orden judicial en la UTI, en la perspectiva de los enfermeros intensivos. Metodología: investigación cualitativa, exploratoria y analítica cuyos participantes fueron 108 enfermeros intensivistas, actuantes en UTI en las regiones sudeste y sur de Brasil y que afirmaron haber vivido situaciones de internación por orden judicial. Los datos fueron recolectados por un cuestionario, en el período entre abril de 2015 a agosto de 2016 y analizados de acuerdo con el Análisis de Contenido. Resultados: se analizaron las categorías aumento por la demanda por el servicio de UTI y falta de estructura institucional y en la región de la residencia del paciente. Conclusiones: la internación por orden judicial, en muchos casos, es el único medio del paciente tener sus derechos garantizados.

Descriptores: Enfermería; Unidades de Cuidados Intensivos; Judicialización de la Salud; Ética; Hospitalización. 


\section{INTRODUÇÂO}

No ano de 2010, os leitos de Unidades de Terapia Intensiva (UTI) adulto no Brasil eram de 21.798. Já, no ano de 2015, ocorreu um aumento de $19 \%$ totalizando 27.060 leitos de UTIs adulto, sendo estes leitos distribuídos em 505 municípios. Destes leitos 13.011 são leitos do SUS (Sistema Único de Saúde) e 14,049 são leitos não SUS. Mesmo com este aumento do número de leitos de UTIs adulto no país, a quantidade de leitos no SUS ainda é insuficiente, sobretudo devido à demanda crescente da população pela busca do serviço ${ }^{(1.2)}$.

Ainda, a distribuição dos leitos de UTI é desigual, sendo que a maior parte dos leitos do país estão localizados na região Sudeste. 2 Esta disparidade na distribuição de recursos de saúde significa que as UTIs tendem a ser localizadas mais em áreas urbanas do que rurais. Portanto, nas áreas rurais os serviços especializados são limitados, o que leva a população a procurar os grandes centros pelos serviços especializados.2,3 Este aumento pela procura do serviço ocorre, também, devido a mudança no cenário demográfico. Segundo estudos ${ }^{(2-6)}$, o perfil populacional tem se modificado, por meio do aumento da expectativa de vida e das condições crônicas de saúde, como: diabetes, hipertensão, doenças coronárias, obesidade. Neste caso, em algum momento da vida, esta população irá precisar de cuidados especializados devido à agudização do quadro da doença( ${ }^{(4,6-8)}$.

Nos casos, no qual os municípios e União não apresentam leitos disponíveis, os familiares possuem o direito de entrar na justiça por um leito de UTI. Nesta perspectiva, as internações por decisões judiciais acontecem quando o município ou - Estado não possui condições ou meios de garantir uma assistência adequada ao paciente grave ou gravíssimo, em situações de urgência/emergência, que representam risco à vida deste individuo. Nesta circunstância, ciente da gravidade do seu familiar e da necessidade de um leito em outro centro, a família recorre ao Ministério Público ou ao seu advogado para proceder ou deferir uma liminar. Esta é uma ação contra o município e o Estado encaminhada à Central de Leitos do Estado, buscando um leito de UTI ${ }^{(1,9)}$.

A internação por ordem judicial em UTIs é uma temática que necessita ser mais amplamente discutida, levando-se em conta seus dilemas e problemas éticos e sócio-econômicos. Alguns estudos ${ }^{(9-1)}$ encontrados relatam que essa internação é o fator de maior relevância nas ações judiciais em saúde requeridas no Brasil, especialmente pelas pessoas de baixa renda, que não tem acesso a vagas em UTIs do SUS.

Constitui-se como objetivo desse estudo analisar os fatores desencadeadores da internação por ordem judicial na UTI, na perspectiva dos enfermeiros intensivistas.

\section{METODOLOGIA}

\section{Tipo de Estudo}

Pesquisa qualitativa, exploratória e analítica.

\section{Participantes da Pesquisa}

A população do estudo totalizou 451 enfermeiros intensivistas que atuam em UTI adulto das regiões sudeste e sul do Brasil e que participaram dos eventos de enfermagem promovidos pela ABENTI - Associação Brasileira de Terapia Intensiva e pela Associação de Medicina Intensiva Brasileira (AMIB). Estes eventos acontecem com abrangência nacional e regional, de modo frequente e sistemático.

Os critérios para a seleção dos participantes da pesquisa foram: atuar com enfermeiro em UTI das regiões sudeste ou sul do Brasil e afirmar ter vivenciado a situação de internação por ordem judicial em seu contexto de trabalho; trabalhar na UTI há mais de um ano e, ainda, não ter respondido ao questionário em evento anterior. Neste caso, 108 participantes vivenciaram e descreveram a situação de internação por ordem judicial na UTI. Os critérios de exclusão dos participantes limitaram-se à falta de disponibilidade ou de interesse em participar da pesquisa, a não devolução dos questionários ou sua devolução em branco.

\section{Coleta dos Dados}

Os dados foram coletados no período entre abril de 2015 e agosto de 2016. E, o instrumento de coleta de dados continha uma parte inicial de caracterização sociodemográfica dos participantes, contendo dados como: idade, sexo, tempo de formação, estado em que trabalha, cursos realizados e titulação, vínculo empregatício, tempo de atuação, carga horária semanal, informação acerca existência Comissão Ética na instituição e de reuniões na unidade de trabalho. Além disso, foi inserida uma questão aberta acerca da vivência do enfermeiro com a inviabilidade de leito de UTI e com a Internação por Ordem Judicial. Os participantes foram identificados pela letra $\mathrm{P}$ e pela numeração sequencial ( $\mathrm{Pl}$ a P108), conforme a ordem de resposta ao questionário e da descrição da situação vivenciada.

\section{Análise dos Dados}

Procedeu-se a Análise de Conteúdo dos discursos dos participantes segundo Bardin ${ }^{(12)}$.

\section{Procedimentos Éticos}

O projeto de pesquisa foi inserido na Plataforma Brasil e aprovado pelo Comitê de Ética e Pesquisa (CEP) da Universidade Federal de Santa Catarina (UFSC), observando- 
se as recomendações da Resolução 466/12 do Conselho Nacional de Saúde, em três de outubro de 2014 com o Parecer número 863.112. Os participantes que aceitaram participar da pesquisa assinaram o Termo de Consentimento Livre e Esclarecido.

\section{RESULTADOS}

Dentre os resultados, foram encontradas duas categorias: "Aumento pela demanda pelo serviço de UTI" e "Falta de estrutura institucional e na região da residência do paciente".

$\mathrm{Na}$ categoria "Aumento pela demanda pelo serviço de UTI", estão presentes os elementos que evidenciam a preocupação dos enfermeiros intensivistas com o aumento pela demanda de leitos, provocados principalmente pela transição das características populacionais em que se observa o rápido envelhecimento e acometimento por doenças crônicas. Verificou-se também como fator desencadeador da internação por ordem judicial a necessidade de realização de procedimentos invasivos: "Leito era reservado para PCR e foi ocupado por paciente internação ordem judicial e realmente a internação era indicada - paciente precisava de um melhor tratamento" (P72).

Nas falas dos enfermeiros, a demanda crescente por leitos de UTI muitas vezes ocorre devido à necessidade de realização de procedimentos cirúrgicos urgentes e que necessitam de cuidados intensivos no pós-operatório ou necessita da internação para a realização de um procedimento especializado: "AUTI em que trabalho atualmente tem realizado cirurgias cardiacas pediátricas, por este motivo, solicitações de vagas no serviço estão aumentando." (P9) “Paciente aguardando vaga para o CTI há vários dias na unidade de urgência e emergência e familiares acionaram a justiça" (P35). "Paciente oncológico, dor intensa, com metástase e a família solicitou internamento em UTI geral para morte assistida e o parecer do júri foi positivo a família" (P58).

Outro aspecto importante apontado pelos enfermeiros intensivistas foi a real necessidade da vaga do leito de UTI: "A paciente era considerada pela equipe de saúde como terminal, sendo indicado cuidados paliativos, e, portanto sem indicações de ocupar leitos de terapia intensiva; afastando - a do conforto familiar e incentivando a eutanásia. A família não aceitou a situação e forçou a internação por ordem judicial. Outro paciente com real necessidade de terapia intensiva aguardou o próximo leito disponível na sala de emergência" (P25). "Lembro de 2 internações juntas e a necessidade então de alta de 2 pacientes, na noite, para proporcionar as vagas. Porém, os pacientes com ordem judicial não eram pacientes com necessidade de terapia intensiva" (P94). "Paciente idosa, sem real indicação de internação em UTI, mas família se sentia mais segura" (P104). "Por mais de 1 vez recebemos determinação da justiça para receber pacientes sem indicação, ou mesmo sem a vaga"(P107). “Um paciente sequelado devido a uma Paraplegia, a família entrou com uma liminar na justiça onde permaneceu na UTI, por mais de um ano até chegar a óbito por infecções recorrentes" (P71). "Idoso com quadro estável, internado há alguns na emergência do hospital, familiares estavam pressionando equipe médica quanto à necessidade de internação na UTI, médicos da emergência orientaram aos familiares a irem ao Ministério Público e assim foi lavrado a ordem de internação judicial. A UTI teve que dar alta precoce para algum paciente e obedecer a ordem judicial. Não era o paciente que mais necessitava daquela vaga na UTI pois existiam pacientes indicações mais ponderantes na situação" (P6l).

E, a problemática se intensifica quando são considerados outros aspectos para além da necessidade de internação: "Paciente jovem politraumatizado. O hospital tem muito cuidado (exagero) com qualquer conduta com este paciente (retarda condutas, realiza superinvestimentos, retarda alta), há um "receio" da família e ações da mesma, por parte de profissionais e hospital (familia influente)" (P39)

$\mathrm{Na}$ segunda categoria são abordados aspectos da falta de estrutura local/regional para atender os pacientes de localidades que não possuem UTIs e a falta de estrutura da instituição hospitalar para atender as necessidades das familias. "Pacientes provenientes do interior do estado e falta de estrutura e condições de atendimento no local de origem" (P47). "Pct do HPS, politrauma que obteve ordem judicial o pedido da cidade que ele morava, pois não tinha leito CTI quando deu entrada no HPS fez primeiros socorros e logo foi transferido p/ outro hospital" (P49). "Sim, trabalho em um pronto socorro de alta complexidade, onde ficamos com pacientes graves sem a estrutura necessária, pois os leitos de UTI do município muitas vezes estão lotados. As internações por ordem judicial são comuns em nossa unidade" (P81). "Falta de leitos e estrutura na cidade vizinha" (P51). "Paciente trauma sem vaga UTI no interior vem com ordem judicial, pois necessitava de cuidados intensivos" (P66).

Ainda, com relação a essa categoria, sinaliza-se que em alguns hospitais há um déficit nos recursos disponiveis para tratamento intensivo, sendo necessária a transferência para hospitais com melhores estruturas de UTI. Nem sempre o hospital para qual o paciente será transferido é o adequado para a sua situação de doença, sendo necessária a transferência para o mais apropriado: "Já recebemos também pacientes vindos de outras unidades de saúde por falta de equipamento e equipe especializada em Cirurgia Cardiaca" (P86). "Paciente esperando dias na upa com necessidade de 
leito em UTI. A advogada entrou em contato para saber de havia leito pois tinha mandato judicial. Porém paciente fora do perfil da unidade, sem estrutura adequada para atendimento. Orientei procurar o hospital de referência da rede para esse caso" (P97).

Verificou-se que em alguns casos a demora na internação resulta na impossibilidade de atendimento qualificado, pois muitas vezes as condições apresentadas pelos pacientes passam a ser irreversíveis: "Paciente com sindrome de Fournier, onde no tratamento a paciente era encaminhada ao CC para realização dos curativos todos os dias, infelizmente veio a falecer" (45). "Paciente precisava de leito UTI chegou proveniente de uma UTI pronto socorro por ordem judicial com várias complicações já oriundas tratamento insuficiente e com várias sequelas (principalmente cuidados de enfermagem)" (P68). "Idosa interna CTI após vídeo que evidenciava cuidado inadequado na unidade internação" (P46).

\section{DISCUSSÃO}

A procura por serviços especializados leva a um número significativo na busca serviços de UTI, excedendo o número de leitos disponibilizados pelo SUS. Logo, a ausência de leitos de UTI é fator predominante para que a população busque seus direitos por meio da justiça. Segundo estudo8, nos últimos anos o principal bem judicializado tem sido o acesso a leitos de UTI, sendo $66 \%$ das demandas judiciais, o que corrobora com as falas dos enfermeiros que vivenciam esta realidade em seu cotidiano(4.11)

A alta demanda por leitos de UTI provoca filas nas centrais de regulação de leitos de UTI, com impacto no fluxo (6). A internação de pacientes por ordem judicial é realizada em muitos casos quando o paciente encontra-se criticamente enfermo, quando há necessidade de monitoração intensiva, quando em coma, com necessidade de intervenção imediata em caso de agravo, com sobrevida reduzida, ou ainda em casos de cuidado individualizado(10).

A necessidade de leitos de UTI especializados devese à importância de um cuidado individualizado, o que pode acarretar a necessidade de equipamentos, profissionais especializados para o cuidado. Logo, analisa-se que, ainda que o serviço seja especializado, muitas vezes o número de enfermeiros por paciente é desproporcional, o que inviabiliza uma assistência adequada(13).

A falta de funcionários e leitos de UTI para atender toda uma população também é um dos grandes problemas encontrados em estudos recentes sobre esta temática. Segundo os autores, a taxa de ocupação de leito versus a relação de enfermeiro é relevante para a assistência clínica, mas também é uma ferramenta essencial em relação à gestão, que estão relacionados com a eficiência e os custos dos serviços ${ }^{(3,13)}$. Neste sentido, atrela-se uma necessária reflexão acerca das possibilidades de cuidado das instituições, considerando o compromisso com a cidadania, os conflitos com a realidade em relação ao que é proposto pela lei orgânica da saúde, que preconiza a equidade, universalidade do cuidado. Neste caso, sobreleva-se a questão da preocupação da enfermagem com as demandas de saúde e complexidades dos serviços, objetivando subsidiar melhores práticas para o cuidado de forma integral ${ }^{(14)}$.

A internação por ordem judicial em muitos casos ocorre devido à falta de estrutura na região/local de moradia do paciente. Há cidades em que o hospital não possui UTI sendo necessária transferência para outra localidade, na qual, há o serviço disponível(5). Devido à ausência de leitos de UTI e a necessidade de se cumprir os processos de internação por ordem judicial, os pacientes muitas vezes são transferidos para outros hospitais. Logo, as transferências interhospitalares ocorrem quando um paciente é transferido de um hospital para outro, no qual há um melhor suporte para a condição de saúde do paciente ${ }^{(15,16)}$. E, nos casos em que ocorre a transferência inter-hospitalar é necessário uma equipe muito bem preparada para realização do transporte, para que este seja seguro e que ocorra o mínimo de danos ao paciente ${ }^{(15)}$. Ainda, um problema que emerge é, justamente, a localidade do hospital para onde o paciente irá ser transferido. Ou seja, em muitos casos a família não aceita a transferência, pois o hospital é longe do local de residência da mesma, o que dificulta para esta se manter em outra cidade ${ }^{(16)}$

Percebem-se enfrentamentos éticos vivenciados pelos enfermeiros intensivistas quando esses têm que pensar em toda a dinâmica por trás da recusa de aceitação de um paciente de um hospital para o outro. Em muitos casos, esta negação pode estar associada a uma unidade que não está preparada para receber o paciente, ou ainda não ter suporte de profissionais para o atendimento. Isso suscita refletir acerca da importância da gestão em saúde, que abrange a promoção da assistência e cuidados em saúde de alta qualidade, por meio do gerenciamento de pessoal, controle de insumos, adequação da área física, controle tecnológico (8).

O pedido de abertura de novos leitos, nos leva a refletir sobre a disparidade de distribuição de leitos de UTI pelo país, na qual, a grande maioria dos leitos encontra-se em áreas urbanas. No Brasil, a distribuição de leitos se faz desigual também em relação às regionais, na qual a região Sudeste apresenta a maioria dos leitos de UTI adulto do país ${ }^{(1,2)}$.

A questão do gerenciamento da abertura de novos leitos também é crucial, pois a alta demanda é um desafio significativo para o enfermeiro gestor. Há necessidade de planejamento da unidade, dos recursos humanos, serviços, equipamentos ${ }^{(3.4)}$. O uso adequado da UTI pode salvar muitas 
vidas. Neste sentido, os recursos devem atender à demanda de qualificação das UTIs e não somente para a abertura de novos leitos ${ }^{(7)}$.

Outro fator desencadeador da internação por ordem judicial apontada pelos participantes como desencadeador do aumento pela demanda de serviços de UTI, é o uso indevido do leito de UTI, por meio de encaminhamentos inadequados, como por exemplo pacientes estáveis e sem a necessidade de cuidados intensivos. Portanto, há necessidade de efetiva triagem que justifique a internação e estabeleça o possivel benefício esperado para o paciente com a internação em leito de $\mathrm{UTI}^{(3)}$.

\section{Limitações do estudo}

Sinaliza-se como limitação do estudo a realização da pesquisa nas regiões Sul e Sudeste, o que pode não representar as múltiplas possibilidades de expressão e de vivências dos enfermeiros diante das situações de internação por ordem judicial em outras regiões do Brasil.

\section{Contribuições do estudo para a prática}

Conhecer os motivos das internações por ordem judicial e a realidade do cotidiano dos enfermeiros intensivistas é essencial para operacionalizar mudanças que qualifiquem o atendimento nas UTIs e, também, entender os mecanismos que conduzem à necessidade da judicialização da saúde. A perspectiva é a efetivação dos direitos dos cidadãos a um atendimento igualitário e universal.

\section{CONCLUSÕES}

Os enfermeiros intensivistas apontam que alguns fatores são importantes para desencadear a internação por ordem judicial e que estes fatores estão intimamente ligados com a realidade de mudança de cenário demográfico. É possivel perceber também diante das falas dos enfermeiros que eles têm passado por dilemas éticos importantes em seu cotidiano de trabalho, no qual se deparam com situações que demandam uma tomada de decisão, por vezes, moralmente questionáveis.

\section{Contribuição dos autores}

Concepção e/ou desenho, análise e interpretação dos dados, redação do artigo, revisão crítica, revisão final: Cintia Iara Oliveira, Mara Ambrosina de Oliveira Vargas, Edison Devos Barlem, Sueli Dias de Araújo, Débora Feijó Vieira, Daniela Cardozo.

Agradecimento: a presente pesquisa é oriunda do projeto financiado pelo Edital Universal 2014 CNPq, cujo título é "Internação por ordem judicial: o exercício da advocacia do paciente pelos enfermeiros intensivistas". 


\section{REFERÊNCIAS}

1. Vargas MAO, Peter E, Luz KR, Ramos FRS, Barlem E, Viana RAPP. Judicialização da saúde e direito do paciente ao atendimento qualificado na terapia intensiva. In: Vargas MAO, Nascimento ERP. (Orgs). PROENF - Programa de Atualização em Enfermagem: Terapia Intensiva. Artmed Panamericana; 2018; 1(4). p. 9-42.

2. Conselho Federal de Medicina. Leitos de UTI no Brasil. Conselho Federal de Medicina [Internet]. 2015. [cited 2017 Dez 28]. Available from: http://portal.cfm.org.br/images/PDF/leitosuti_tipodeuti.pdf

3. Gordon K, Allorto N, Wise R. Analysis of referrals and triage patterns in a South African metropolitan adult intensive care service. SAMJ [Internet]. 2015 Jun [cited 2017 Aug 17]; 105(6): 49195. Available from: http://www.scielo.org.za/scielo.php?script=sci_ arttextEpid=SO256-95742015000600025.

4. Matlakala MC, Bezuidenhout MC, Botha ADH. Challenges encountered by critical care unit managers in the large intensive care units. Curationis 2014 [cited 2017 Aug 16]; 37(1):1146. Available from: http://dx.doi. org/10.4102/curationis. v37il.1146.

5. Fowler RA, Abdeimalik P, Wood G, Foster D, Gibney N, et al. Critical care capacity in Canada: results of a national cross-sectional study. Critical Care [Internet]. 2015 [cited 2017 Aug 17]; 19:133. Available from: https://ccforum.biomedcentral.com/articles/10.1186/s13054-0150852-6.

6. Goldwasser RS , Lobo MC, Arruda EF, Angelo SA, Silva JRL, Salles AA David CM,et al. Dificuldades de acesso e estimativas de leitos públicos para unidades de terapia intensiva no estado do Rio de Janeiro. Rev Saúde Pública [Internet]. 2016 [cited 2017 Aug 17]; 50(19):1-10. Available from: http://www.scielo.br/pdf/rsp/v50/pt_0034-8910rsp-S1518-87872016050005997.pdf

7. Machado FR. All in a Day's Work - Equity vs. Equality at a Public ICU in Brazil. N Engl J Med 375;25. Dec 22, 2016.2420-1.

8. Novaretti MCZ, Quitério LM, Santos EV. Gestão em unidades de terapia intensiva brasileiras: estudo bibliométrico dos últimos 10 anos. Revista de administração hospitalar e inovação em saúde [Internet]. 2015 [cited 2017 Aug 18]; 12(4):16-33. Available from: http://revistas. face.ufmg.br/index.php/rahis/article/view/2623/1633.
9. Vargas MAO, Ramos FRS, Schneider DG, Schneider N, Santos AC, Leal SMC. Internação por ordem judicial: dilemas éticos vivenciados por enfermeiros. Revista Gaúcha de Enfermagem [Internet]. 2013; [cited 2017 May 23]; 34(1): 119-25. Available from: http://www.scielo.br/ pdf/rgenf/v34nl/15.pdf.

10. Diniz D, Machado TRC, P J. A judicialização da saúde no Distrito Federal, Brasil. Ciênc. saúde coletiva [Internet]. 2014 Feb [cited 2017 Aug 19]: 19(2): 591-98. Available from: http://www.scielo.br/scielo. php?script=sci_arttext\&pid=S1413-81232014000200591\&lng=en http://dx.doi.org/10.1590/1413-81232014192.23072012.

11. Ramos RS, Gomes AMT, Oliveira DC, Marques SC, Spindola T, Nogueira VPF. O acesso às ações e serviços do Sistema Único de Saúde na perspectiva da judicialização. Rev. Latino-Am. Enferm. 2016; 24:e2797.

12. Bardin L. Análise de conteúdo. São Paulo: Edições 70, 2016.

13. De Vos MLG, Veer SNV, Wouterse B, Graafmans WC, Peek N, Keizer NF, et al. A multifaceted feedback strategy alone does not improve the adherence to organizational guideline-based standards: a cluster randomized trial in intensive care. Implementation Science [Internet]. 2015 [cited 2017 Aug 17]; 10:95. Available from: http:// implementationscience.biomedcentral.com/articles/10.1186/s13012015-0285-2.

14. André SR, Rodrigues ILA, Nogueira LMV, Santos MNA, Morais TM. Responsabilidade técnica em enfermagem: conhecendo sua importância para o exercicio profissional. Enferm. Foco [Internet]. 2019 [cited 2019 Apr 25]; 10(1): 46-51. Available from: http://revista. cofen.gov.br/index.php/enfermagem/article/view/1512/492

15. Conselho Nacional de Justiça. Judicialização da saúde no Brasil: dados e experiência. Coordenadores: Felipe Dutra Asensi e Roseni Pinheiro. Brasilia, 2015. 142 p.

16. Droogh JM, Smit M, Absalom AR, Ligtenberg JJM, Zijlstra JG. Transferring the critically ill patient: are we there yet?. Critical Care [Internet]. 2015 [cited 2017 Aug 17]; 19:62. Available from: https://www. ncbi.nlm.nih.gov/pmc/articles/PMC4335540/. 